\title{
Concentration statistics for transport in random media
}

\author{
Marco Dentz* \\ Institute of Environmental Assessment and Water Research (IDFA-CSIC), 08034 Barcelona, Spain \\ Diogo Bolster \\ Department of Geotechnical Engineering and Geosciences, Technical University of Catalonia (UPC), 08034 Barcelona, Spain \\ Tanguy Le Borgne \\ Geosciences Rennes, UMR 6118, CNRS, Université de Rennes 1, 3500 Rennes, France \\ (Received 9 March 2009; revised manuscript received 30 April 2009; published 14 July 2009)
}

\begin{abstract}
We study the ensemble statistics of the particle density in a random medium whose mean transport dynamics describes a continuous time random walk. Starting from a Langevin equation for the particle motion in a single disorder realization, we derive evolution equations for the $n$-point moments of concentration by coarse graining and ensemble averaging the microscale transport problem. The governing equations describe multidimensional continuous time random walks whose waiting time distribution is given in terms of the disorder distribution. We find that the concentration is not self-averaging even for normal mean behavior. The relative concentration variance for anomalous is larger than for normal mean behavior. These results may have some impact on risk and extreme value analysis in stochastic dynamic systems.
\end{abstract}

DOI: 10.1103/PhysRevE.80.010101

PACS number(s): 05.40.-a, 02.50.Ey, 05.10.Gg, 05.60.Cd

In the frame of effective approaches for transport in disordered media, it is of paramount importance to quantify the reliability of the predicted mean behavior. This implies the quantification of the ensemble statistics of concentration and more specifically the self-averaging behavior of the particle density as measured by its relative ensemble variance. Effective models can be obtained by phenomenological considerations or derived using stochastic models for the spatiotemporal fluctuations of the disordered medium. In the latter case it is possible to relate the characteristics of the disorder distribution to the parameters and constitutive equations of the effective transport model and quantify the fluctuations of the state variables and, ideally, their full statistical distribution.

We consider transport in disordered media, whose mean behavior can be described by continuous time random walks (CTRWs) [1,2]. CTRW is an ensemble averaged transport theory [3] that is ubiquitously used in many fields ranging from solid-state physics to financial physics to biology to groundwater hydrology $[2,4,5]$. It has been used to model a series of dynamical phenomena including the diffusion of carriers in disordered media [1], turbulent diffusion [6], anomalous diffusion in intermittent chaotic systems [7], anomalous dispersion of light in disordered optical materials [8], and non-Gaussian contaminant transport in geological media $[9,10]$.

Klafter and Silbey [3] showed that CTRW can be obtained by ensemble averaging over a certain not further specified disorder distribution. Eisenberg et al. [11] addressed the problem of asymptotic concentration fluctuations for an unbiased CTRW which describes anomalous diffusive mean behavior.

Here the aim is to quantify the ensemble statistics for

\footnotetext{
*marco.dentz@upc.edu
}

transport in random media which on average is given by a CTRW. Transport in a single realization of such a medium can be described by the Langevin equations [12],

$$
d \mathbf{x}(s)=\mathbf{v}[\mathbf{x}(s)] d s+\sqrt{2 D d s} \boldsymbol{\xi}, \quad d t(s)=\theta(s) d s .
$$

The drift $\mathbf{v}(\mathbf{x})$ is induced by a deterministic external field, $D$ is the molecular diffusion coefficient, and $\boldsymbol{\xi}$ is a Gaussian random vector characterized by zero mean and unit variance. The deterministic drift $\mathbf{v}(\mathbf{x})$ is characterized by the typical value $v_{0}$. The small scale medium fluctuations are reflected in the time increment $\theta(s) d s>0$. The heterogeneity coefficient $\theta(s)$ is modeled as a stationary random variable. The fluctuations of the random medium are characterized by the heterogeneity scale $l$. Equation (1) describes the movement of a particle in a random environment under a deterministic external drift force and subject to white noise. The typical drift $v_{0}$, the diffusion coefficient $D$, and the heterogeneity scale $l$ define the Péclet number $\mathrm{Pe}=v_{0} l / D$. The initial conditions for Eq. (1) are $\mathbf{x}(0)=0$ and $t\left(0^{-}\right)=0 ; t(s)$ denotes the time when the particle leaves $\mathbf{x}(s)$.

Note that the particle position $\mathbf{x}(t)$ is an implicit function of time, $\mathbf{x}(t)=\mathbf{x}[s(t)]$. Thus, the particle distribution $c(\mathbf{x}, t)$ in a single realization of $\theta(s)$ is given by

$$
c(\mathbf{x}, t)=\langle\delta\{\mathbf{x}-\mathbf{x}[s(t)]\}\rangle,
$$

where the angular brackets denote the white-noise average. The $n$th ensemble moment of the particle distribution then is given by

$$
\overline{c^{n}}(\mathbf{x}, t)=\overline{\langle\delta\{\mathbf{x}-\mathbf{x}[s(t)]\}\rangle^{n}},
$$

where the overbar denotes the disorder average over the ensemble $\{\theta(s)\}$. Expression (3) can be written in terms of $t(s)$ as 


$$
\overline{c^{n}}(\mathbf{x}, t)=\int_{0}^{\infty} d s\langle\delta[\mathbf{x}-\mathbf{x}(s)]\rangle^{n} \overline{\theta(s) \delta[t-t(s)]},
$$

where we used that $\delta[f(t)]=[d f / d t]^{-1} \delta\left[t-t_{0}\right]$ with $t_{0}$ as the only zero of $f(t)$.

In the following we consider the more general $n$-point moments of concentration

$$
\phi_{n}(\hat{\mathbf{x}}, t)=\int_{0}^{\infty} d s \prod_{i=1}^{n}\left\langle\delta\left[\mathbf{x}^{(i)}-\mathbf{x}^{(i)}(s)\right]\right\rangle \times \overline{\theta(s) \delta[t-t(s)]} .
$$

We define the $n d$-dimensional position vector $\hat{\mathbf{x}}$ $=\left[\mathbf{x}^{(1)^{\prime}}, \ldots, \mathbf{x}^{(n)^{\prime}}\right]^{\prime}$, where the prime denotes the transpose. Each of the $\mathbf{x}^{(i)}(s)$ satisfies the Langevin Eq. (1). The $n$th moment of the particle distribution in terms of the $n$-point density [Eq. (5)] is $\overline{c^{n}}(\mathbf{x}, t)=\phi_{n}\left[\mathbf{x}^{(1)}=\mathbf{x}, \ldots, \mathbf{x}^{(n)}=\mathbf{x}, t\right]$. Specifically, the mean density is given by $\bar{c}(\mathbf{x}, t)=\phi_{1}(\mathbf{x}, t)$; the density variance is given by $\sigma_{c}^{2}(\mathbf{x}, t)=\phi_{2}(\mathbf{x}, \mathbf{x}, t)-\phi_{1}(\mathbf{x}, t)^{2}$. Note that Eq. (5) is not the $n$-point displacement density as studied in Ref. [13], for example. In the notation used in this Rapid Communication the $n$-point displacement density reads as $\overline{c\left(\mathbf{x}_{1}, t_{1} ; \ldots ; \mathbf{x}_{N}, t_{N}\right)}$.

In order to perform the ensemble average in Eq. (5) we need to specify the statistics of $\theta(s)$ and the spatial organization of the random medium. As outlined above, the medium fluctuations are characterized by a typical (microscopic) heterogeneity scale, that is, the correlation scale of the medium fluctuations, given by $l$. For illustration, we consider only a single heterogeneity scale. In general, the spatial organization of the medium can be more complex and characterized by a hierarchy of length scales. We focus on an observation scale much larger than $l$ on which the medium can be considered uncorrelated so that $\theta(s)$ is completely characterized by its single variable distribution $\mathcal{P}(\theta)$. We choose the heterogeneity scale $l$ as the new spatial support scale, which introduces the typical advection time $\tau_{v}=l / v_{0}$ as the new temporal support scale. Note that coarse graining is a key step here because it allows us to take into account the information on the medium organization.

We now discretize the operational time $s$ in terms of $\tau_{v}$. That is, we set $s=N \tau_{v}$ and identify $\mathbf{x}^{(i)}(s) \equiv \mathbf{x}_{N}^{(i)}, \theta(s) \equiv \theta_{N}$, and

$$
\int_{0}^{s} d s^{\prime} \theta\left(s^{\prime}\right) \equiv \sum_{n=0}^{N} \tau_{v} \theta_{n}
$$

The sum starts at $n=0$, which implies that the particle leaves the initial position $\mathbf{x}_{0}=\mathbf{0}$ at the random time $t_{0}=\tau_{v} \theta_{0}$. The discretized version of Eq. (5) is now given by

$$
\begin{aligned}
\phi_{n}(\hat{\mathbf{x}}, t)= & \tau_{v} \sum_{N=0}^{\infty} \prod_{i=1}^{n}\left\langle\delta\left[\mathbf{x}^{(i)}-\mathbf{x}_{N}^{(i)}\right]\right\rangle \\
& \times \int_{0}^{t} d t^{\prime} \overline{\theta_{N} \delta\left(t-t^{\prime}-\tau_{v} \theta_{N}\right)} \delta\left[t^{\prime}-\sum_{n=0}^{N-1} \theta_{n} \tau_{v}\right] .
\end{aligned}
$$

For observation times much larger than $\tau_{v}$ we approximate

$$
\overline{\theta \delta\left(t-\tau_{v} \theta\right)} \approx \tau_{v}^{-1} \int_{t / \tau_{v}}^{\infty} d \theta \mathcal{P}(\theta) .
$$

Upon defining the transition time $\tau=\theta \tau_{v}$ and its distribution $\psi(\tau)$ as

$$
\psi(\tau)=\tau_{v}^{-1} \mathcal{P}\left(\tau / \tau_{v}\right)
$$

expression (7) for the $n$-point density is

$$
\phi_{n}(\hat{\mathbf{x}}, t)=\int_{0}^{t} d t^{\prime}\left[1-\int_{0}^{t-t^{\prime}} d t^{\prime \prime} \psi\left(t^{\prime \prime}\right)\right] R\left(\hat{\mathbf{x}}, t^{\prime}\right),
$$

where we define

$$
R(\hat{\mathbf{x}}, t)=\sum_{N=0}^{\infty}\left\langle\delta\left[\hat{\mathbf{x}}-\hat{\mathbf{x}}_{N}\right]\right\rangle \overline{\delta\left(t-t_{N}\right)} .
$$

The discrete $(n d+1)$-dimensional process $\left(\hat{\mathbf{x}}_{N}, t_{N}\right)$ satisfies

$$
\begin{gathered}
\hat{\mathbf{x}}_{N}=\hat{\mathbf{x}}_{N-1}+\hat{\mathbf{v}}\left(\hat{\mathbf{x}}_{N-1}\right) \tau_{v}+\sqrt{2 D \tau_{v}} \hat{\boldsymbol{\xi}}_{N} \\
t_{N}=t_{N-1}+\tau_{N-1},
\end{gathered}
$$

where the $\hat{\boldsymbol{\xi}}_{N}$ is an $n d$-dimensional Gaussian distributed independent random vector with zero mean and unit variance, the $n d$-dimensional drift is given by $\hat{\mathbf{v}}=\left(\mathbf{v}^{\prime}, \ldots, \mathbf{v}^{\prime}\right)^{\prime}$. The independent time increments are distributed according to $\psi(\tau)$. Note that $t_{N}$ denotes the time at which the particle reaches the position $\hat{\mathbf{x}}_{N}$. The initial conditions are $\hat{\mathbf{x}}_{0}=\mathbf{0}$ and $t_{0}=0$. Equations (10), (11), (12a), and (12b) define a classical decoupled CTRW in the presence of a deterministic external drift force field.

The generalized master equation corresponding to the CTRW [Eqs. (10), (11), (12a), and (12b)] then is given by [1]

$$
\begin{aligned}
\frac{\partial \phi_{n}(\hat{\mathbf{x}}, t)}{\partial t}= & \int_{0}^{t} d t \int d \hat{\mathbf{x}}^{\prime} \tau_{v}^{-1} \Phi\left(\hat{\mathbf{x}} \mid \hat{\mathbf{x}}^{\prime}\right) \\
& \times M\left(t-t^{\prime}\right)\left[\phi_{n}\left(\hat{\mathbf{x}}^{\prime}, t^{\prime}\right)-\phi_{n}(\hat{\mathbf{x}}, t)\right],
\end{aligned}
$$

where the transition probability $\Phi\left(\hat{\mathbf{x}} \mid \hat{\mathbf{x}}^{\prime}\right)$ is given by $\Phi\left(\hat{\mathbf{x}} \mid \hat{\mathbf{x}}^{\prime}\right)=\left.\left\langle\delta\left[\hat{\mathbf{x}}-\hat{\mathbf{x}}_{N}\right]\right\rangle\right|_{\hat{\mathbf{x}}_{N-1}=\hat{\mathbf{x}}^{\prime}}[14]$. The memory function $M(t)$ reads in Laplace space as

$$
M^{*}(\lambda)=\frac{\lambda \tau_{v} \psi^{*}(\lambda)}{1-\psi^{*}(\lambda)} .
$$

The Laplace transform is denoted here by an asterisk, the Laplace variable is $\lambda$.

A Kramers-Moyal expansion [14] of Eq. (13) up to second order gives the following generalized Fokker-Planck equation for $\phi_{n}(\hat{\mathbf{x}}, t)$ :

$$
\begin{aligned}
\frac{\partial \phi_{n}(\hat{\mathbf{x}}, t)}{\partial t}= & -[\hat{\boldsymbol{\nabla}} \cdot \hat{\mathbf{v}}(\hat{\mathbf{x}})-\hat{\boldsymbol{\nabla}}: \hat{\nabla} \hat{\mathbf{D}}(\hat{\mathbf{x}})] \\
& \times \int_{0}^{t} d t^{\prime} M\left(t-t^{\prime}\right) \phi_{n}\left(\hat{\mathbf{x}}, t^{\prime}\right),
\end{aligned}
$$

where the colon denotes a tensor product. The dispersion tensor $\hat{\mathbf{D}}(\hat{\mathbf{x}})$ is given by 


$$
\hat{\mathbf{D}}(\hat{\mathbf{x}})=\mathbb{1}_{n d} D+\frac{\hat{\mathbf{v}}(\hat{\mathbf{x}}): \hat{\mathbf{v}}(\hat{\mathbf{x}})}{2} \tau_{v},
$$

with $\mathbb{1}_{n d}$ the $n d$-dimensional identity matrix. For $n=1$, we denote $\hat{\mathbf{D}}$ by $\mathbf{D}$.

For the exponential heterogeneity distribution $\mathcal{P}(\theta)=\exp (-\theta)$ the memory function $M(t)$ reduces to $M(t)=\delta(t)$, and Eq. (15) reduces to a Fokker-Planck equation, which means that mean transport is normal. For illustration in the following, we consider a constant external drift force $\mathbf{v}(\mathbf{x})=\mathbf{v}=$ const, which allows us to obtain closed form expressions for the concentration statistics. The solutions to Eq. (15) for an exponential disorder distribution then are given by the $n d$-dimensional Gaussian pulse

$$
\varphi_{n d}(\hat{\mathbf{x}}, t, \hat{\mathbf{v}}, \hat{\mathbf{D}})=\frac{\exp \left[-(\hat{\mathbf{x}}-\hat{\mathbf{v}} t) \hat{\mathbf{D}}^{-1}(\hat{\mathbf{x}}-\hat{\mathbf{v}} t) / 4 t\right]}{\left[(4 \pi t)^{n d} \operatorname{det} \hat{\mathbf{D}}\right]^{1 / 2}},
$$

with $\hat{\mathbf{D}}^{-1}$ as the inverse of $\hat{\mathbf{D}}$. Specifically, the mean concentration $\bar{c}(\mathbf{x}, t)=\phi_{1}(\mathbf{x}, t)$ is given by a Gaussian pulse, that is, transport is normal.

In the limit of $\mathrm{Pe}=0$, i.e., in the force-free case, the variance is zero, which means that the concentration is exactly self-averaging [11]. In the presence of an external field, this is different. Aligning the one direction of the coordinate system with the direction of the drift $\mathbf{v}$ so that $v_{i}=\delta_{i 1} v$, we obtain the explicit expression for the concentration variance

$$
\sigma_{c}^{2}=\bar{c}^{2}\left\{\frac{D_{11} \exp \left[\frac{\left(x_{1}-v t\right)^{2} v^{2} \tau_{v}}{4 t D_{11}\left(D+v^{2} \tau_{v}\right)}\right]}{\sqrt{\left(D+v^{2} \tau_{v}\right) D}}-1\right\} .
$$

Note that this implies that even in the case of normal mean transport, the relative variance $\sigma_{c}^{2} / \bar{c}^{2}$ increases exponentially for increasing distance from the center of mass of the average distribution. In the limit of large times, the relative variance diverges exponentially with time. This means that in contrast to the force-free case, the relative concentration variance is always finite or, in other words, the concentration is a non-self-averaging observable. That is, the ensemble mean concentration is not (asymptotically) equal to the coarse grained or spatially averaged concentration in a single medium realization. Similar non-self-averaging behavior has been observed in Ref. [15] for the first passage time distribution in a one-dimensional disordered medium.

For arbitrary disorder distributions, the solution of Eq. (15) can be expressed in terms of the Laplace transform of Eq. (17) as

$$
\phi_{n}^{*}(\hat{\mathbf{x}}, \lambda)=\varphi_{n d}^{*}\left[\hat{\mathbf{x}}, \lambda, \hat{\mathbf{v}} M^{*}(\lambda), \hat{\mathbf{D}} M^{*}(\lambda)\right] .
$$

Thus, the general Laplace space solutions for the mean and mean-squared concentrations in $d=1$ dimension are

$$
\vec{c}^{*}(x, \lambda)=\exp \left[x v /\left(2 D_{11}\right)\right] \times \frac{\exp \left(-\frac{|x| v}{2 D_{11}} \sqrt{1+4 \frac{\lambda D_{11}}{M^{*}(\lambda) v^{2}}}\right)}{M^{*}(\lambda) v \sqrt{1+4 \frac{\lambda D_{11}}{M^{*}(\lambda) v^{2}}}},
$$

$$
\begin{aligned}
\bar{c}^{2^{*}}(x, \lambda)= & \frac{\exp \left[x v /\left(D+v^{2} \tau_{v}\right)\right]}{2 \pi} \\
& \times \frac{K_{0}\left(\frac{|x| v}{D+v^{2} \tau_{v}} \sqrt{1+2 \lambda \frac{D+v^{2} \tau_{v}}{v^{2} M^{*}(\lambda)}}\right)}{M^{*}(\lambda) \sqrt{\left(D+v^{2} \tau_{v}\right) D}} .
\end{aligned}
$$

In the following we consider the concentration variance for non-Fickian mean transport as observed for the truncated power-law distribution [16]

$$
\mathcal{P}(\theta) \propto \exp \left[-\left(\frac{1}{\theta-1}+\frac{\theta-1}{\theta_{c}}\right)\right](\theta-1)^{-1-\beta},
$$

with $\theta \geq 1$. In the time regime $\tau_{v} \ll t \ll \tau_{v} \theta_{c}$ transport is anomalous that is for $0<\beta<2$ the mean and variance of the particle trajectory scale anomalously with time (e.g., [17]). For times $t \gg \tau_{v} \theta_{c}$ the mean behavior asymptotes toward normal [18] and the concentration variance is asymptotically given by Eq. (18). These results are compared to Monte Carlo simulations based on the numerical solution of the Langevin equation

$$
d \mathbf{x}(t)=\frac{\mathbf{v} d t}{R[\mathbf{x}(t)]}+\sqrt{\frac{2 D d t}{R[\mathbf{x}(t)]}} \xi,
$$

which is equivalent to Eq. (1) for a constant drift force. This can be seen by performing the subordination transformation $d t=R[\mathbf{x}(s)] d s$ (e.g., [19]), approximating $\mathbf{x}(s) \approx \mathbf{v} s$ and identifying $\theta(s) \equiv R(\mathbf{v} s)$.

Figure 1 shows the squared mean concentration and concentration variances obtained by Laplace inversion of Eqs. (20) and (21) using Eq. (22) for two different cut-off values $\left(\theta_{c}=10,10^{3}\right), \beta=3 / 2$ at $t=10^{3} \tau_{v}$ in $d=1$. Notably, the uncertainty of concentration increases as mean transport becomes more anomalous, i.e., for increasing cut-off value $\theta_{c}$. The dots denote the Monte Carlo results obtained by numerical solution of Eq. (23) for $10^{3}$ particles in $2 \times 10^{4}$ disorder realizations characterized by the truncated power-law distribution [Eq. (22)]. The simulated behavior agrees well with the model predictions. In both cases the absolute variance increases as the concentration values increase. Large concentration values are spatially more localized than small values and thus subject to larger uncertainty. For anomalous mean transport, which is characterized by a spatial backward tail [Fig. 1(b)], this behavior is amplified as the spatial distribution of large concentration values is broader than for normal mean transport.

In conclusion, we have presented a method that allows for the determination of the full concentration statistics for transport in random media whose mean behavior can be modeled as a CTRW. We have studied the concentration variance for normal and anomalous mean transport behavior and have found that the concentration distribution is in general not self-averaging in the presence of an external field. Experimentally determined concentration values are usually obtained as spatial or temporal averages. Our results imply that the point values of concentration can significantly deviate 

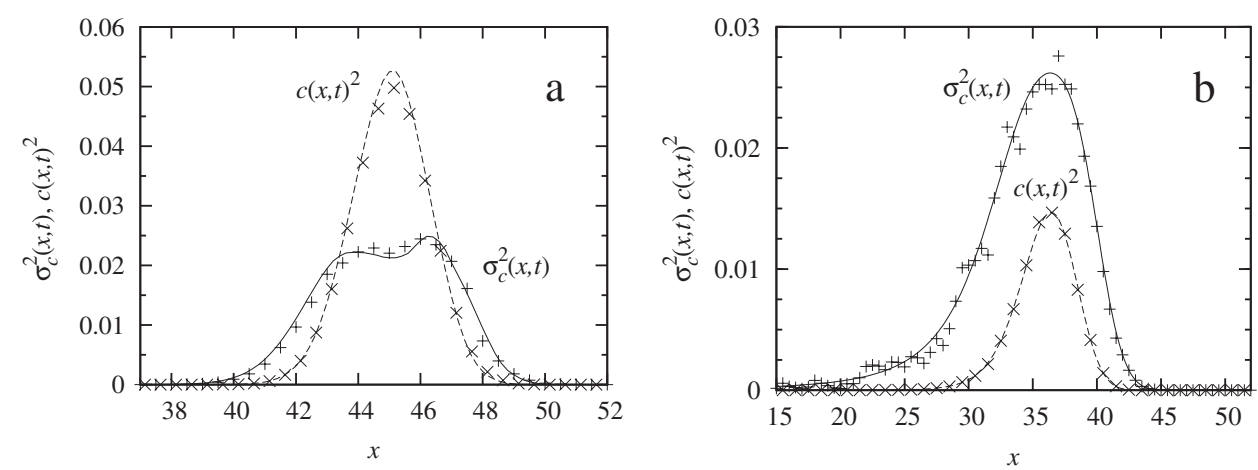

FIG. 1. Concentration variances (solid lines) and squared mean concentrations (dashed lines) in $d=1$ dimension obtained by inverse Laplace transform of Eqs. (20) and (21) for the disorder distribution [Eq. (22)] with $\beta=3 / 2$ and the cut-off parameters (a) $\theta_{c}=10$ and (b) $\theta_{c}=10^{3}$. The Péclet number is $\mathrm{Pe}=10$ and the observation time $t=10^{3} \tau_{v}$. The corresponding Monte Carlo results for the squared mean concentrations $(X)$ and concentration variances $(+)$ are obtained by numerical solution of the Langevin Eq. (23) for the movements of $10^{3}$ particles in each of $2 \times 10^{4}$ disorder realizations. The relative variance of concentration increases as the mean transport behavior becomes more anomalous.

from the measured mean. Specifically, we find that the relative concentration variance for anomalous mean transport behavior is larger than the one for normal mean transport. This implies that for non-Fickian mean transport as frequently observed in real systems, the uncertainty of the observed concentration values can be significant, which is of importance for extreme value studies and risk analysis in disordered systems.
[1] H. Scher and M. Lax, Phys. Rev. B 7, 4491 (1973).

[2] R. Metzler and J. Klafter, Phys. Rep. 339, 1 (2000).

[3] J. Klafter and R. Silbey, Phys. Rev. Lett. 44, 55 (1980).

[4] J. P. Bouchaud and A. Georges, Phys. Rep. 195, 127 (1990).

[5] B. Berkowitz, A. Cortis, M. Dentz, and H. Scher, Rev. Geophys. 44, RG2003 (2006).

[6] M. F. Shlesinger, B. J. West, and J. Klafter, Phys. Rev. Lett. 58, 1100 (1987).

[7] T. Geisel and S. Thomae, Phys. Rev. Lett. 52, 1936 (1984).

[8] P. Barthelemy, J. Bertolotti, and D. S. Wiersma, Nature (London) 453, 495 (2008).

[9] B. Berkowitz and H. Scher, Phys. Rev. Lett. 79, 4038 (1997).

[10] T. Le Borgne, M. Dentz, and J. Carrera, Phys. Rev. Lett. 101, 090601 (2008).

[11] E. Eisenberg, S. Havlin, and G. H. Weiss, Phys. Rev. Lett. 72,
2827 (1994).

[12] H. C. Fogedby, Phys. Rev. E 50, 1657 (1994).

[13] A. Baule and R. Friedrich, EPL 77, 10002 (2007).

[14] H. Risken, The Fokker-Planck Equation (Springer, Heidelberg, NY, 1996).

[15] M. Kawasaki, T. Odagaki, and K. W. Kehr, Phys. Rev. B 61, 5839 (2000).

[16] M. Dentz and A. Castro, Geophys. Res. Lett. 36, L03403 (2009).

[17] G. Margolin and B. Berkowitz, Phys. Rev. E 65, 031101 (2002).

[18] M. Dentz, A. Cortis, H. Scher, and B. Berkowitz, Adv. Water Resour. 27, 155 (2004).

[19] W. Feller, An Introduction to Probability Theory and Its Applications (Wiley, New York, 1957), Vol. 2. 